$201811^{\text {th }}$ IFIP Wireless and Mobile Networking Conference (WMNC)

September 3-5, 2018, Prague, Czech Republic

The Proceedings of the 2018

$11^{\text {th }}$ IFIP Wireless and Mobile

Networking Conference (WMNC) 
Volume editor: Dusan Maga

The Proceedings of the $201811^{\text {th }}$ IFIP Wireless and Mobile Networking Conference (WMNC)

Processed by: Czech Technical University in Prague, Czech Republic

Number of Pages: 140

$1^{\text {st }}$ Edition

Contact: Dusan Maga, Czech Technical University in Prague, Technicka 2, 16627 Prague, Czech Republic

Tel.: (+420) 22435 4021, e-mail: dusan.maga@cvut.cz

(C) IFIP International Federation for Information Processing 2018

This work is subject to copyright. All rights are reserved by IFIP, whether the whole or part of the material is concerned, specifically the rights of translation, reprinting, reuse of illustrations, recitation, broadcasting, reproduction on microfilms or in any other physical way, and transmission or information storage and retrieval, electronic adaption, computer software, or by similar or dissimilar methodology now know or hereafter developed. 


\section{Volume editor:}

Dusan Maga

\section{Full Name of Publication:}

The Proceedings of the $201811^{\text {th }}$ IFIP Wireless and Mobile Networking Conference (WMNC)

Each paper has been reviewed by 3 independent reviewers. The responsibility for the content and language of each paper rests solely on its author(s). First edition, 2018.

ISBN: 978-3-903176-03-4 (USB, Part Number: CFP1895K-USB)

(ISBN: 978-3-903176-04-1 (XPLORE COMPLIANT, Part Number: CFP1895K-ART); online ISSN 2473-3644)

\section{Technical support, inquiries:}

Dusan Maga, Czech Technical University in Prague, Technicka 2, 16627 Prague, Czech Republic Tel.: (+420) 224354021

e-mail: dusan.maga@cvut.cz 


\section{$21081^{\text {th }}$ IFIP Wireless and Mobile Networking Conference (WMNC) Chairs and Committees}

General Chairs

- Miroslav Voznak, VSB - Technical University Ostrava, Czech Republic

- Robert Bestak, Czech Technical University in Prague, Czech Republic

\section{Honorary Chair}

- Vaclav Snasel, VSB - Technical University Ostrava, Czech Republic

\section{Technical Program Chairs}

- Floriano De Rango, University of Calabria, Italy

- Ray-Guang Cheng, National Taiwan University of Science, Taiwan

\section{Publicity Chairs}

- Jakub Klemsa, Czech Technical University in Prague, Czech Republic

\section{Publication Chairs}

- Dusan Maga, Czech Technical University in Prague, Czech Republic

\section{Web Chair}

- Marek Nevosad, Czech Technical University in Prague, Czech Republic

- Jan Rozhon, VSB - Technical University Ostrava, Czech Republic

\section{Organizing Committee}

- Terezia Nemcova, Czech Technical University in Prague, Czech Republic

- David Blagodarny, Czech Technical University in Prague, Czech Republic

- Iyad Khuder, Czech Technical University in Prague, Czech Republic

- Zagroz Aziz, Czech Technical University in Prague, Czech Republic

- Pavol Partila, VSB - Technical University Ostrava, Czech Republic

- Jaromir Tovarek, VSB - Technical University Ostrava, Czech Republic

- Veronika Mandincova, VSB - Technical University Ostrava, Czech Republic

Steering Committee

- Pedro Cuenca, Universidad de Castilla-La Mancha, Spain

- Robert Bestak, Czech Technical University in Prague, Czech Republic

- Giuseppe Bianchi, Università degli Studi di Roma - Tor Vergata, Italy

- Hannes Frey, University of Koblenz-Landau, Germany

- Mario Gerla, UCLA Computer Science Department, US

- Zoubir Mammeri, Paul Sabatier University, France

- Edmundo Monteiro, Coimbra University, Portugal

- George C. Polyzos, Athens University of Economics and Business, Greece

- Guy Pujolle, Pierre et Marie Curie University Paris 6, France

- Jaime Lloret, Universidad Politécnica de Valencia, Spain

- Pascal Lorenz, University of Haute Alsace, France 
- Adam Wolisz, Technische Universität Berlin, Germany

- Jozef Wozniak (SC Chair), Gdansk University of Technology, Poland

\section{Technical Program Committee}

- S. Agrawal, Delhi Technological University (DTU) Formerly Delhi College of Engineering (DCE), India

- Wessam Ajib, Université du Québec à Montréal, Canada

- Rastislav Bencel,Slovak University of Technology, Slovakia

- Zinaida Benenson, University of Erlangen-Nuremberg, Germany

- Christos Bouras, University of Patras CTI\&P-Diophantus, Greece

- Raffaele Bruno, IIT-CNR, Italy

- Claudia Campolo, University Mediterranea of Reggio Calabria, Italy

- Eduardo Cerqueira, Federal University of Para \& UCLA, Brazil

- Edward Chlebus, Illinois Institute of Technology, USA

- György Dán, KTH Royal Institute of Technology, Sweden

- Peppino Fazio, University of Calabria, Italy

- Mario Freire, University of Beira Interior, Portugal

- José Ramón Gállego, University of Zaragoza, Spain

- Ivan Ganchev, University of Limerick, Ireland / University of Plovdiv "Paisii Hilendarski", Bulgaria

- Yacine Ghamri-Doudane, University of la Rochelle, France

- Sonia Heemstra de Groot, Eindhoven Technical University, The Netherlands

- Pavol Helebrandt, Slovak University of Technology, Slovakia

- Jiri Hosek, Brno University of Technology, Czech Republic

- Jerzy Konorski, Gdansk University of Technology, Poland

- Hakkı Gökhan İlk, Ankara University, Turkey

- Ivan Kotuliak, Slovak University of Technology, Slovakia

- Francine Krief, University of Bordeaux, France

- Zbynek Kocur, Czech Technical University in Prague, Czech Republic

- Sławomir Kukliński, Warsaw University of Technology, Poland

- Pavel Lafata, Czech Technical University in Prague, Czech Republic

- Shou-Chih Lo, National Dong Hwa University, Taiwan

- Zoubir Mammeri, Paul Sabatier University, France

- Dora Maros, Obuda University, Hungary

- Miralem Mehic, University of Sarajevo, Bosnia and Herzegovina

- Manar Mohaisen, Korea University of Technology and Education, Korea

- Edmundo Monteiro, University of Coimbra, Portugal

- Sasa Mrdovic, University of Sarajevo, Bosnia and Herzegovina

- Marek Natkaniec, AGH University of Science and Technology, Poland

- Sarat Patra, National Institute of Technology, Rourkela, India

- Paulo Pereira, INESC-ID, Portugal

- Thomas Plagemann, University of Oslo, Norway

- Ana Pont, Universitat Politécnica de València, Spain

- Alessandro Redondi, Politecnico di Milano, Italy

- Michal Ries, Slovak University of Technology, Slovakia

- K. Martin Sagayam, Karunya Unniversity, India 
- Oriol Sallent, Universitat Politècnica de Catalunya, Spain

- Alireza Shahrabi, Glasgow Caledonian University, United Kingdom (Great Britain)

- YeQiong Song, LORIA - University of Lorraine - France, France

- Yutaka Takahashi, Kyoto University, Japan

- Homero Toral-Cruz, University of Quintana Roo, Mexico

- Manabu Tsukada, the Univerrsity of Tokyo, Japan

- Ardian Ulvan, University of Lampung, Indonesia

- Bernd Wolfinger, University of Hamburg, Germany

- Jozef Wozniak, Gdansk University of Technology, Poland

- Yong Xiao, University of Arizona, USA

- Sherali Zeadally, University of Kentucky, USA

- Yi Zhang, Trinity College Dublin, Ireland 


\section{Message from the General Chairs}

It is our honour and pleasure to welcome you to the $11^{\text {th }}$ IFIP/IEEE Wireless and Mobile Networking Conference (WMNC 2018) which is being held in the campus of one of the oldest technical universities in Central Europe, at the Czech Technical University in Prague, Czech Republic, on September 3-5, 2018.

The objective of this edition of WMNC 2018 is to attract innovative research works in the areas of mobile and wireless networks, services, applications and mobile computing. This goal has been reached and we would especially like to thank the Technical Program Committee Co-chairs, Floriano De Rango, Ray-Guang Cheng, who efficiently organised the review process and composed an outstanding technical program.

This event is organized by two Czech universities, the VSB - Technical University of Ostrava and the Czech Technical University in Prague. We have prepared five paper sessions and three keynote speeches. Besides technical conference program, we hope, many of the attendees also take time to enjoy Prague and visit major cultural monuments in the marvelous city.

All this would not be possible without the hard and enthusiastic work of a number of people who contributed to make WMNC 2018 a successful conference. We would like to thank all of them, from the Technical Committee chairs and members across the Publication and Workshop chairs and the Local Organizing Committee to the authors and all staff that helped organize local matters. Great thanks are also dedicated to the Steering Committee of WMNC 2018.

Last but not least, we would very much like to encourage current and future authors to continue working in this exciting direction of research and participate in forums similar to this conference to promote explorative engineering and exchange of scientific knowledge and experience. 


\section{Technical Program Committee Chairs' Message}

It is a great pleasure to welcome you to the proceedings of WMNC 2018. This international conference WMNC 2018 received a technical sponsoring from Czechoslovakia Section of IEEE and was approved as IFIP Conference by IFIP TC6 Working Group 6.8.

The technical program this year consists of selected regular papers divided into five sessions within three days accompanied by three keynote speeches opening individual conference days. We would like to thank all authors submitting papers in WMNC 2018. We received 33 paper submissions, and we accepted 16 regular papers, and 3 short papers, according to reviewers' recommendations. Papers came from authors from Europe, Asia, and North America. The TPC was formed by 52 researchers from 26 different countries. All papers underwent a thorough review process, with each paper receiving three reviews at least.

As regards topics of the WMNC 2018, various domains of wireless communications are covered in the conference proceedings; however, several topics predominate over others, especially mobile AdHoc networks, localization, and Internet of things.

We would like to express our thanks to all the members of the TPC and to all the additional reviewers for all their work that made possible the preparation of the technical program of WMNC 2018. Also, we have to appreciate the support and contribution of General Chairs Miroslav Voznak and Robert Bestak, and last but not least, we would like to thank all participants for attending the conference that we believe will be interesting and challenging. 
Eduardo Soares; Ana C Aguiar; Pedro Brandao; Rui Prior: Service for MANET Node Self-Configuration and Communication

Samradnyee Pawar; Sandeep Thorat; Durgesh Kshirsagar: Evaluating MANET Uncertainty

Analysis Framework

Muhammad Omer Farooq; Dirk Pesch: ERPL: An Enhanced Peer-to-Peer Routing Mechanism for Low-Power and Lossy Networks

Vincenzo Inzillo; Floriano De Rango; Alfonso Ariza Quintana: Mobility Beamforming Prediction and a Round

Robin Scheduling in a Directional MAC for MANET.

Davide Giovanelli; Elisabetta Farella: RSSI or Time-of-flight for Bluetooth Low Energy Based Localization?

An Experimental Evaluation

Marcelo Nogueira de Sousa; Reiner S. Thoma: Sensor Selection in Multipath Scenario with Particle Swarm

Optimization and Ray Tracing Fingerprints

Thomas Tegou; Ilias Kalamaras; Konstantinos Votis; Dimitrios Tzovaras: A Low-Cost Room-Level Indoor Localization System with Easy Setup for Medical Applications

Mevlut T Garip; Peter Reiher; Mario Gerla: BOTVEILLANCE: A Vehicular Botnet Surveillance Attack against Pseudonymous Systems in VANETs

Ali Beydoun; Hamze Haidar Alaeddine; Haidar Elmokdad: New Fast Time Synchronization Method for MIMO-OFDM Systems

Ditthawat Songratthaset; Suwat Pattaramalai: Nonlinear Power Amplifier Characteristic Based on Orthogonal Polynomials Method for FBMC Systems

Christos J Bouras; Stylianos Kokkalis; Anastasia Kollia; Andreas Papazois: Techno-Economic Analysis of MIMO \& DAS in $5 \mathrm{G}$

Jiri Svub; Pavel Stasa; Filip Benes; Lukas Vojtech; Marek Neruda; Tomas Brozek: Autonomous System for UHF RFID Signal Measurement in Industrial Environment

Kristian Kostal; Rastislav Bencel; Michal Ries; Ivan Kotuliak: Performance Evaluation of SDN WLAN Architecture

Selman Kulac; Murat Husnu Sazli; Hakki Gokhan Ilk: External Relaying Based Security Solutions for Wireless Implantable Medical Devices: A Review

Amilcare-Francesco Santamaria; Mauro Tropea; Peppino Fazio; Pierfrancesco Raimondo; Floriano De Rango; Miroslav Voznak: A Decentralized ITS Architecture for Efficient Distribution of Traffic Task Management.........98

Marek Beranek; Ilia Lisunov; Vaclav Vacek: Learning IoT Skills in the Context of Student Projects . .103

Tan N. Nguyen; Phu Tran Tin; Phuong Tran; Hoang Quang Minh Tran; Miroslav Voznak: Power-Splitting Protocol in Power Beacon-assisted Energy Harvesting Full-Duplex Relaying Networks: Performance Analysis .. 109

Carmen Delgado; Sergio Batista; Maria Canales; Jose Ramon Gallego; Jorge Ortin; Matteo Cesana:

An Implementation for Dynamic Application Allocation in Shared Sensor Networks

Floriano De Rango; Amilcare-Francesco Santamaria; Peppino Fazio; Pierfrancesco Raimondo: MDMC:

A WSN Cooperative Protocol for Minimizing the Data Distortion. 


\section{List of Authors}

Aguiar, Ana C.

Alaeddine, Hamze Haidar................................... 61

Batista, Sergio.............................................. 116

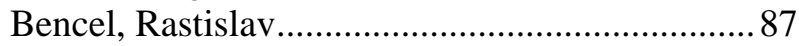

Benes, Filip .............................................. 81

Beranek, Marek............................................... 103

Beydoun, Ali .............................................. 61

Bouras, Christos J. ....................................... 73

Brandao, Pedro .................................................. 1

Brozek, Tomas .................................................. 81

Canales, Maria ................................................ 116

Cesana, Matteo ................................................ 116

De Rango, Floriano.............................25, 98, 124

de Sousa, Marcelo Nogueira ................................ 40

Delgado, Carmen ........................................... 116

Elmokdad, Haidar ............................................... 61

Farella, Elisabetta ............................................. 32

Farooq, Muhammad Omer............................... 17

Fazio, Peppino .....................................98, 124

Gallego, Jose Ramon ................................... 116

Garip, Mevlut T. ..........................................5 53

Gerla, Mario...................................................... 53

Giovanelli, Davide.......................................... 32

Ilk, Hakki Gokhan ......................................... 94

Inzillo, Vincenzo................................................ 25

Kalamaras, Ilias ............................................... 46

Kokkalis, Stylianos ......................................... 73

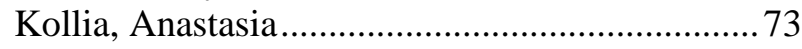

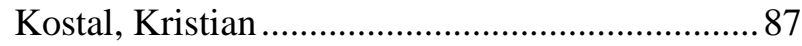

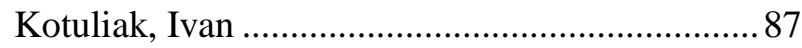

Kshirsagar, Durgesh ...................................... 9

Kulac, Selman.............................................. 94

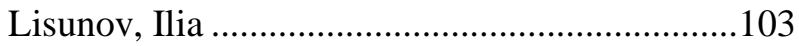

Neruda, Marek..................................................81

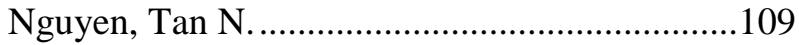

Ortin, Jorge .................................................116

Papazois, Andreas ........................................73

Pattaramalai, Suwat.......................................67

Pawar, Samradnyee .........................................9

Pesch, Dirk .................................................17

Phu, Tran Tin ...................................................109

Prior, Rui ........................................................... 1

Quintana, Alfonso Ariza ..................................25

Raimondo, Pierfrancesco ...........................98, 124

Reiher, Peter...................................................53

Ries, Michal ...................................................87

Santamaria, Amilcare-Francesco ................98, 124

Sazli, Murat Husnu............................................99

Soares, Eduardo................................................. 1

Songratthaset, Ditthawat ................................67

Stasa, Pavel ................................................81

Svub, Jiri ......................................................... 81

Tegou, Thomas................................................46

Thoma, Reiner S...............................................40

Thorat, Sandeep................................................. 9

Tran, Phuong ....................................................109

Tran, Hoang Quang Minh ..................................109

Tropea, Mauro ....................................................98

Tzovaras, Dimitrios...........................................46

Vacek, Vaclav .............................................103

Vojtech, Lukas ................................................81

Votis, Konstantinos .......................................46

Voznak, Miroslav ...................................98, 109 